\title{
Köbner Phenomenon in a Rituximab-Treated Pemphigus Patient: Beware Disease Activity
}

\author{
Soheil Tavakolpour ${ }^{1}$, Hamidreza Mahmoudi ${ }^{1}$, Nika Kianfar ${ }^{1}$, \\ Shayan Dasdar ${ }^{1}$, Maryam Daneshpazhooh ${ }^{1}$
}

1 Autoimmune Bullous Diseases Research Center, Tehran University of Medical Sciences, Tehran, Iran

Key words: pemphigus vulgaris, pemphigus foliaceus, Köbner phenomenon, rituximab, surgery

Citation: Tavakolpour S, Mahmoudi H, Kianfar N, Dasdar S, Daneshpazhooh M. Köbner phenomenon in a rituximab-treated pemphigus patient: beware disease activity. Dermatol Pract Concept. 2019;9(4):320-321. DOI: https://doi.org/10.5826/dpc.0904a20

Accepted: June 26, 2019; Published: October 31, 2019

Copyright: @2019 Tavakolpour et al. This is an open-access article distributed under the terms of the Creative Commons Attribution License, which permits unrestricted use, distribution, and reproduction in any medium, provided the original author and source are credited.

Funding: None.

Competing interests: The authors have no conflicts of interest to disclose.

Authorship: All authors have contributed significantly to this publication.

Corresponding author: Hamidreza Mahmoudi, MD, Autoimmune Bullous Diseases Research Center, Razi Hospital, Vahdate-Eslami Square, 11996, Tehran, Iran. Email: hr_mahmoody@yahoo.com

\section{Introduction}

Pemphigus consists of a group of rare autoimmune blistering diseases that are typically characterized by the appearance of intraepithelial blisters on the skin and/or mucosa. Pemphigus vulgaris (PV) and pemphigus foliaceus (PF) are 2 well-known forms of pemphigus. Although they have distinct clinicopathological features, each of these conditions could switch to the other. The Köbner phenomenon (KP) is described as the appearance of new lesions in the uninvolved skin as a consequence of different kinds of trauma. It has been described in several skin disorders, including psoriasis, vitiligo, lichen planus, and rarely pemphigus [1]. Here we report a patient with previously diagnosed mucocutaneous PV who developed PF after rituximab (RTX) therapy and developed crusted lesions some weeks after surgery for femoral avascular necrosis at the surgical site.

\section{Case Presentation}

A 55-year-old man with an 8-year history of mucocutaneous PV (confirmed with pathological findings and direct immu- nofluorescence) was treated with RTX in February 2017, after his disease had flared. Both anti-desmoglein (anti-Dsg) 1 and anti-Dsg 3 were positive $(>200 \mathrm{U} / \mathrm{mL})$ at the time of infusion. His disease went into remission within 3 months after the last dose of RTX and remained on minimal therapy (5 mg prednisolone daily and $7.5 \mathrm{mg}$ methotrexate weekly) for more than a year. In May 2018 he complained of the development of dry lesions on the chest, consistent with PF. After checking anti-Dsg1/3 titers, we found that anti-Dsg1 was $63 \mathrm{U} / \mathrm{mL}$, while anti-Dsg3 was negative. In June 2018 a surgical procedure was performed in an attempt to treat femoral avascular necrosis. Despite the successful surgery, after 6 weeks new lesions appeared on the surgery site, which seemed to be related to KP (Figure 1, A and B). At the last follow-up in May 2019, the lesions were about to disappear via low-dose steroid treatment (Figure 2, A and B).

\section{Conclusions}

In addition to several suggested triggers for pemphigus, trauma can trigger the onset of new lesions in patients with pemphigus. A variety of procedures from minor skin sur- 


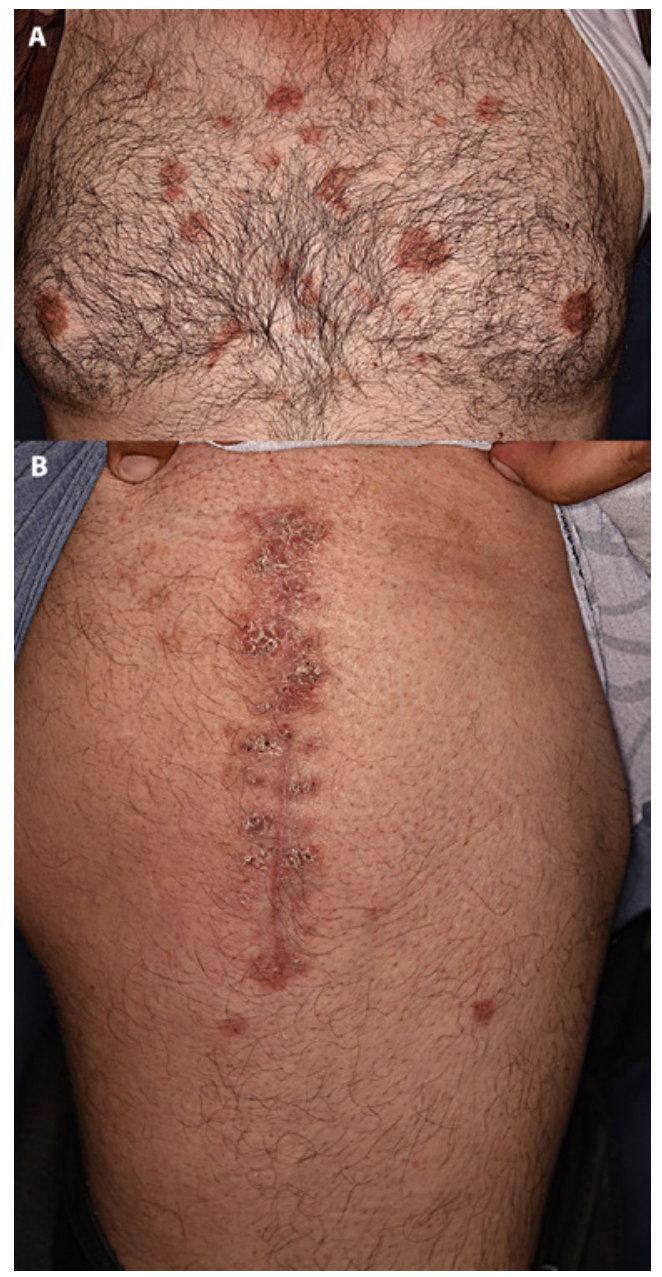

Figure 1. (A) Pemphigus foliaceus lesions on the chest, representing disease activity. (B) New lesions that developed approximately 6 weeks after hip surgery suggest Köbner phenomenon. [Copyright: (C2019 Tavakolpour et al.]

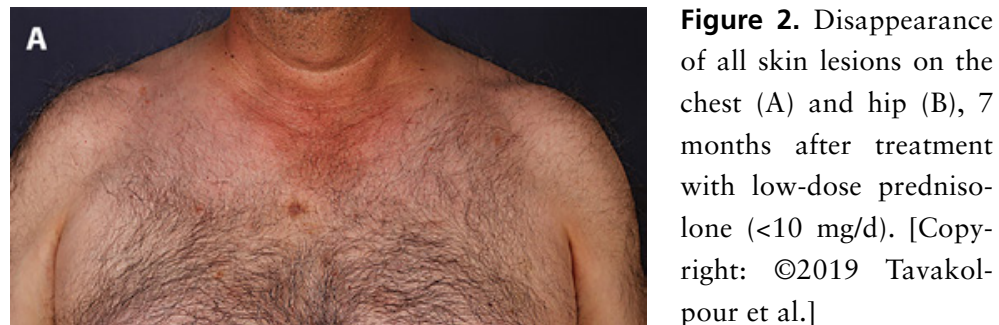

pour et al.] geries to major abdominal and thoracic surgeries have been reported as triggers of $\mathrm{KP}$ in pemphigus. However, the number of reported cases is too small to draw any conclusion about the pathogenesis of KP. The reported mean interval between trauma and the development of lesions was approximately 5 weeks [2]. In our patient, the disease was both clinically and serologically active at the time of surgery, which might be a risk factor for KP.

In general, unnecessary surgery and blunt trauma should be avoided in patients with pemphigus [2]. Necessary procedures should be postponed if possible to a time when the patient is both clinically and serologically in remission, to minimize the risk of KP. This could be achieved 1-3 months after RTX therapy and usually lasts for 6-12 months. How- ever, if the surgery is urgent and patient is not in remission, increasing the dose of corticosteroid could probably prevent $\mathrm{KP}$ or at least mitigate its risk. However, further studies are needed to better understand approaches for preventing KP in patients with pemphigus.

\section{References}

1. Balighi K, Daneshpazhooh M, Azizpour A, et al. Koebner phenomenon in pemphigus vulgaris patients. JAAD Case Rep. 2016;2(5):419-421.

2. Daneshpazhooh M, Fatehnejad M, Rahbar Z, et al. Trauma-induced pemphigus: a case series of 36 patients. J Dtsch Dermatol Ges. 2016;14(2):166-171. 